\title{
Laparoscopic resection of a gastric glomangioma
}

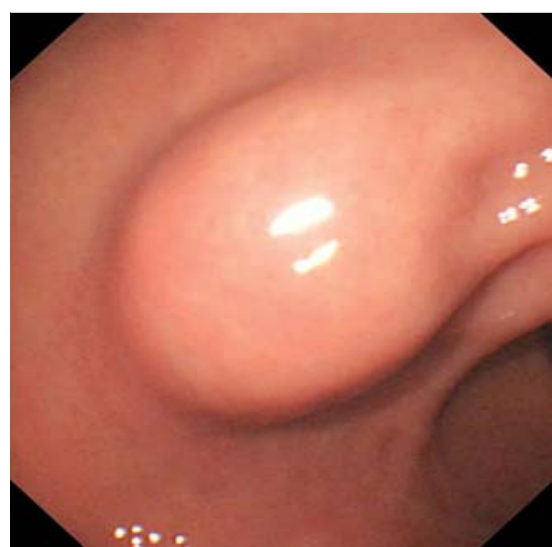

Fig. 1 Endoscopic appearance in a 51-year-old woman with epigastric distension showing a hemispheric bulge in the mucosa of the lesser curvature of the distal gastric body.

A 51-year-old woman underwent gastroscopy because of epigastric distension. The mucosa in the lesser curvature of the distal gastric body showed a hemispheric bulge, with a smooth surface and normal color ( Fig.1). An ultrasound scan showed the presence of a lesion located within the submucosa. It was olive shaped with a medium echo, and the boundary was distinct and smooth with uneven echoes. Multiple hypoechoic lumen-like structures, which typically measured approximately $2 \mathrm{~mm}$, were observed inside the lesion. Color Doppler showed a rich blood flow inside these lumen-like structures ( $\bullet$ Fig. 2 ).

A preliminary diagnosis of gastric hemangioma was made and a laparoscopic wedge resection was performed ( $\bullet$ Fig. 3). Subsequent pathologic examination showed lesions in the submucosa and between the muscles that were consistent with glomangioma ( $\bullet$ Fig.4). Immunohistochemical staining was negative with CD117, CD34, DOG.1, S-100, CgA, p53, and AE1/AE3; weakly positive with Vimentin ( Fig.5) and Syn; moderately positive with SMA; and $\mathrm{Ki}-67$ revealed $5 \%-10 \%$ positivity.

Gastric glomangioma is a rare benign gastric tumor. Most patients show no symptoms, but some do have gastrointestinal bleeding. One of the 32 cases reported by Miettinen et al. showed invasive biologic behavior [1]. In our case, the capsule remained intact after resection and the cells showed no atypical morphology; no recurrence or metastases have occurred within a year of follow-up.

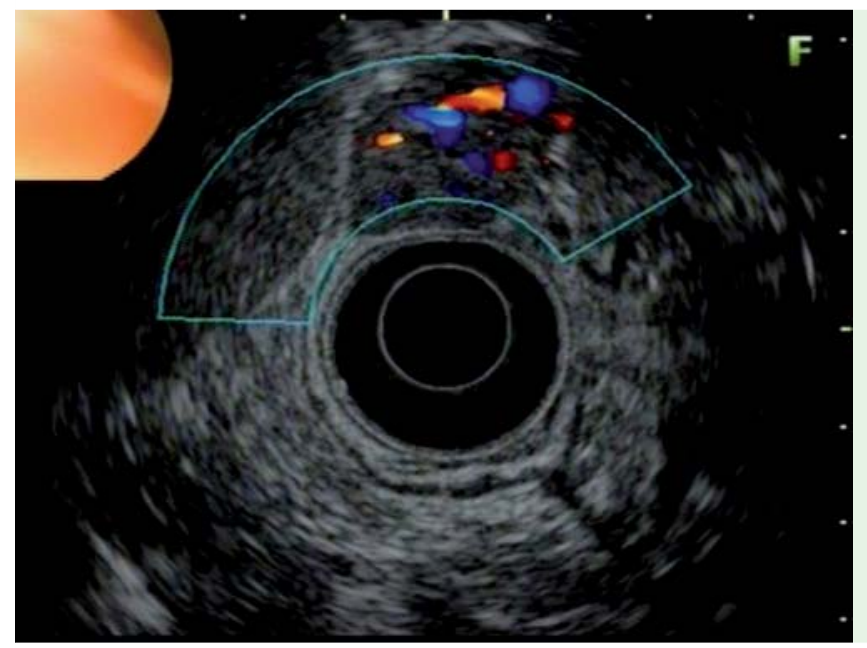

Fig. 2 Color Doppler ultrasound view of the lesion showing rich blood flow within the multiple hypoechoic lumen-like structures that were identified in the lesion.

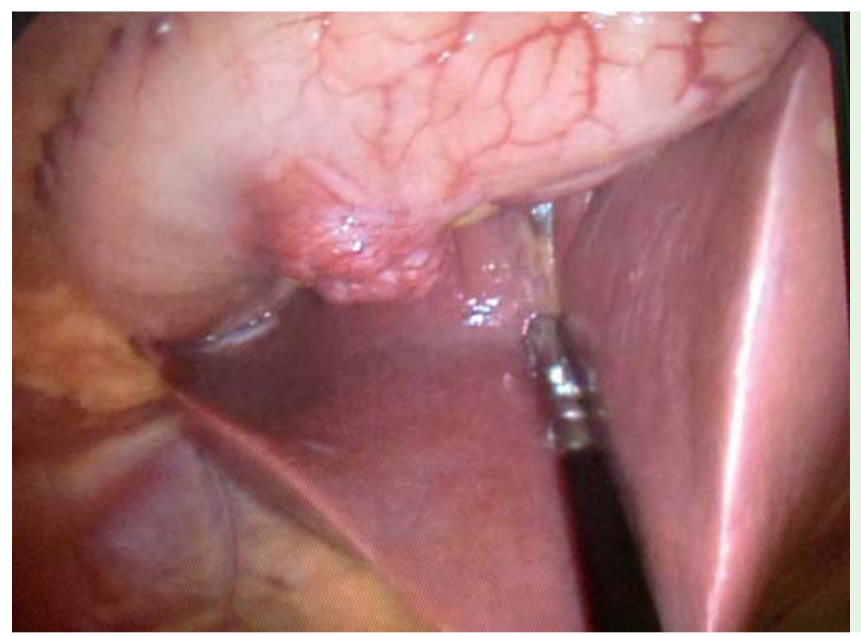

Fig. 3 Appearance during laparoscopic wedge resection of the gastric hemangioma.

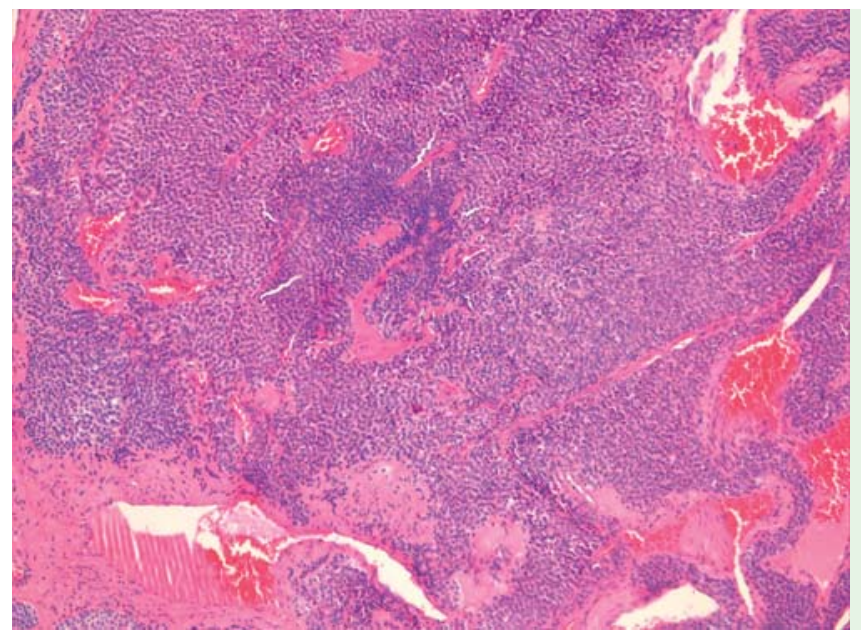

Fig.4 Pathologic appearance of the wedge resection specimen showing lesions located in the submucosa and between the muscles, which were consistent with a glomangioma.

Endoscopy_UCTN_Code_CCL_1AB_2AD_3AF

Competing interests: None

\section{Xiao-jun Zhao, Hang-hong Wang, Jian-qiu Sheng, Na Li}

Department of Gastroenterology, Beijing Military General Hospital, Beijing, China 


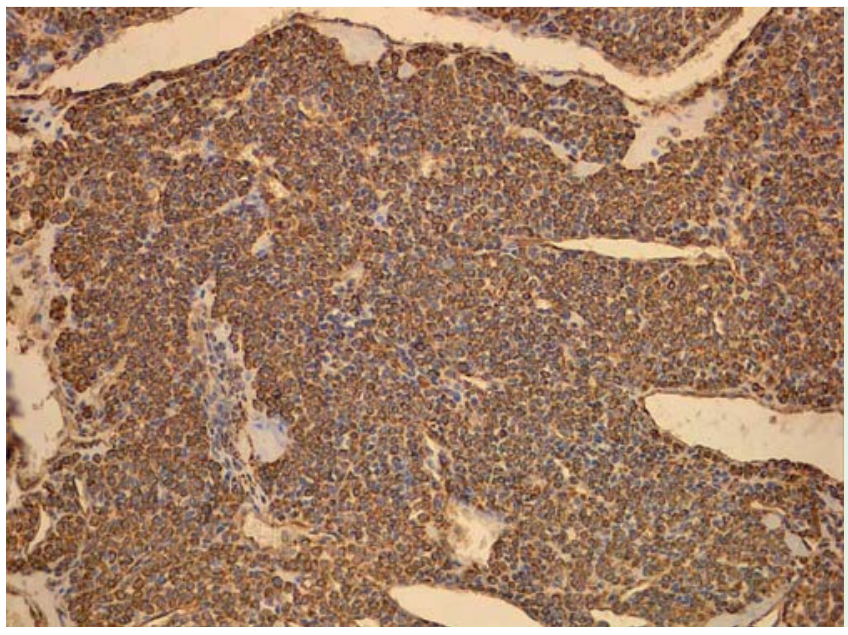

Fig.5 Immunohistochemical staining of the wedge resection specimen showing positivity for Vimentin.

\section{References}

1 Miettinen M, Paal E, Lasota J et al. Gastrointestinal glomus tumors: a clinicopathologic, immunohistochemical, and molecular genetic study of 32 cases. Am J Surg Pathol 2002; 26: $301-311$

\section{Bibliography}

DoI http://dx.doi.org/

10.1055/s-0033-1358927

Endoscopy 2014; 46: E73-E74

(c) Georg Thieme Verlag KG

Stuttgart · New York

ISSN 0013-726X

\section{Corresponding author}

\section{Jian-qiu Sheng, MD}

Department of Gastroenterology Beijing Military General Hospital Nanmenchang 5\#, Dongcheng District Beijing 100700

China

Fax: +86-10-66721299

zhaoxiaojun2@163.com 